\title{
Beam focusing in reflections from flat subwavelength diffraction gratings
}

\author{
Y. C. Cheng, ${ }^{1}$ J. Redondo, ${ }^{2}$ and K. Staliunas ${ }^{1,3}$ \\ ${ }^{1}$ Departament de Fisica i Enginyeria Nuclear, Universitat Politècnica de Catalunya, Colom 11, 08222 Terrassa, Barcelona, Spain \\ ${ }^{2}$ Instituto de Investigación para la Gestión Integrada de Zonas Costeras, Universitat Politècnica de València, Paranimf 1 , \\ 46730 Gandia, València, Spain \\ ${ }^{3}$ Institució Catalana de Recerca i Estudis Avançats (ICREA), Pg. Lluís Companys, 23, 08010 Barcelona, Spain
}

(Received 23 June 2013; published 10 March 2014)

\begin{abstract}
We predict that narrow beams, reflecting from flat subwavelength diffraction gratings, can focus. The effect is shown for the beams of electromagnetic radiation; however, it should be observable for beams of waves of arbitrary nature (microwaves, surface plasmons, and acoustic and mechanical waves). We present analytical estimations of the focusing performance obtained by multiple scattering calculations and demonstrate the focusing effect numerically for an optical system (reflections from an array of dielectric cylinders), using the finite-difference time-domain calculations.
\end{abstract}

DOI: 10.1103/PhysRevA.89.033814

PACS number(s): 42.25.Fx, 42.55.Tv, 42.79.-e

\section{INTRODUCTION}

Diffraction gratings, for both transmission and reflection, are conventionally used in superwavelength regime, i.e., when the grating period $d$ is larger than the wavelength $\lambda$ of the diffracting wave. The angle of the $m$ th-order diffraction component $\beta$ obeys a well-known relation: $|\sin (\beta)-\sin (\alpha)|=$ $m \lambda / d$, where $\alpha$ is the incidence angle. In subwavelength regime $d<\lambda$ neither the first $m=1$ nor the higher $m>1$ diffraction orders appear, and the reflection and transmission of the zero component $m=0$ depend on the averaged characteristics of the grating, as the wave does not distinguish its subwavelength structure. Subwavelength gratings are applied, e.g., for exciting the surface waves: surface plasmons in optics [1], or surface modes of sound waves in acoustics [2], for enhancement of Raman scattering [3], or for reduction of thermo-refractive noise in reflections [4]. Other applications of subwavelength gratings rely on transmission and reflection dependence on polarization and on frequency [5].

All the above applications of subwavelength gratings consider the transmission and reflection of the plane waves, whereas the transformations of the narrow beams reflecting from or transmitting through such gratings have never been considered to the best of our knowledge. In this paper, we predict that the narrow beams, with the width of few wavelengths, can transform in a peculiar and unexpected way reflected from such subwavelength gratings. In particular, the beams can broaden (diffuse) or narrow (antidiffuse) in reflections depending on the beam carrier wave frequency. However, most unexpectedly, the beam wave fronts can obtain additional curvature in reflection, which implies that the reflected beams could focalize.

First, in Sec. II we describe the physical mechanism of the phenomenon basing on simple multiple scattering wave propagation theory, which is a common model for waves of arbitrary nature. Next, in Sec. III we justify the idea by exact numerical calculations in optical systems using the finite difference time domain (FDTD) method, which shows the beam transformations predicted by a semianalytic treatment, and, in particular, proves the main result of the paper: the focusing of the beams in reflection from a flat subwavelength grating. The analytical estimations and the main part of exact numerical calculations are performed for a two-dimensional (2D) model (the wave is propagating in 2D space and reflects from the one-dimensional grating); however, we also generalize the idea for the three-dimensional (3D) cases (the beam is propagating in 3D space and reflecting from 2D gratings of different symmetries) in Sec. IV. Finally, in Sec. V we show that the effects of focusing can be enhanced when the beams reflect from several layers of subwavelength grating.

We note that we consider the grating as a perfectly periodic structure, in contrast to recent studies [6,7], where the subwavelength gratings with the local modulation period or local modulation amplitude varying across the grating are shown to provide the far field focusing in transmission [6] as well as in reflection [7]. Such modulation evidently introduces an optical axis, which breaks the lateral invariance. The grating in our case is laterally invariant (on a scale larger than the grating period).

\section{MULTIPLE SCATTERING ANALYSIS}

The idea is illustrated in Fig. 1. A conventional approach of wave reflection from a grating considers the grating as an array of secondary sources for the reflecting wave. For simplicity of analytical treatment we consider a periodic array of point scatterers; however, in FDTD numerical studies, the scatterers are circles of dielectric material (the cylindrical rods in the 3D case) of finite radii. For subwavelength gratings the character of reflection in the lowest order is analogous to the reflection from a plane interface: the reflected beam propagates as emitted by an image source positioned symmetrically on the opposite side of the grating [Fig. 1(a)].

This simple approach of substitution of the grating by a flat semitransparent mirror does not take into account a possible secondary scattering, when the waves reflect subsequently from two neighboring point scatterers, or generally also from more remote scatterers. If the direct reflection coefficient is $s$ in terms of the amplitude, then the secondary reflection is of the order of magnitude of $O\left(s^{2}\right)$. The image source now is a superposition of several images: the primary image source emitting the wave with the amplitude $s$, and the secondary images, laterally shifted by the grating period and emitting the 
(a)

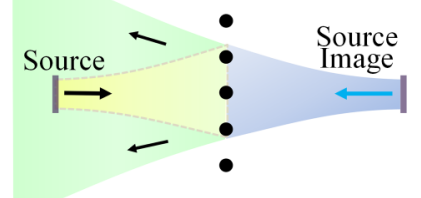

(c)

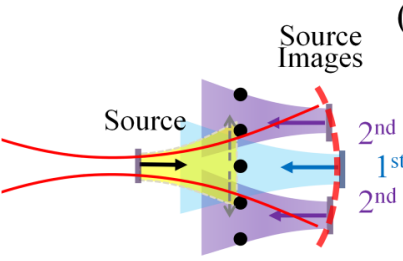

(b)

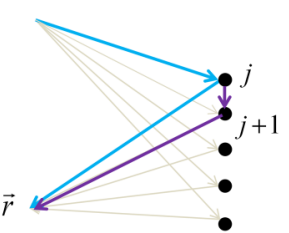

(d)

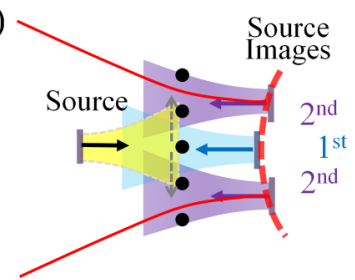

FIG. 1. (Color online) Illustrations of the focusing effect: (a) direct (primary) reflection of the beam from subwavelength gratings and the primary image of source; (b) multiple scattering scheme for calculations of primary and secondary reflections, where the laterally shifted secondary images appear due to secondary reflections (only scattering from nearest neighbors is considered); the case (c) of focusing and (d) of defocusing.

waves of amplitude of $O\left(s^{2}\right)$. As Figs. 1(c) and 1(d) illustrate, the image sources will be diffused or antidiffused due to this secondary scattering, depending on the phase shift between the primary and secondary image sources. The phase of the composed image can become curved so the composed image source can emit the beam with a curved wave front, which implies focusing or defocusing.

Summarizing the above discussion: the image source is expected to be diffused for a zero or for a multiple of a $2 \pi$ phase shift between the primary and secondary image sources, i.e., when the grating period is approximately equal to the wavelength $d \approx \lambda$. For the smaller grating period $d \approx \lambda / 2$, on the other hand, the primary and secondary images sources emit in antiphase. The image source can be then considered as an "antidiffused" source, as the secondary images truncate the fronts of a primary image. Finally, in intermediate cases, the composed image source emits wave with curved wave front. The emitted wave fronts will be convex for $\lambda / 2<d<\lambda$, as the phase of the secondary image source is advanced with respect to the phase of primary source [Fig. 1(c)], and therefore the focalization of reflected beam is expected. The phase shift is represented by a corresponding shift of the secondary image sources along the $z$ axis. For comparison the defocusing case is shown in Fig. 1(d), occurring for, e.g., $\lambda<d<3 \lambda / 2$, and resulting in concave wave fronts of the beam emitted by image sources.

The above discussion is below made rigorous by multiple scattering calculations. Considering primary reflections only, the reflected field is calculated as a sum over all point scatterers: $\sum_{j} s A_{0}\left(\vec{r}_{j}\right) p\left(\vec{r}-\vec{r}_{j}\right)$, where $p\left(\vec{r}-\vec{r}_{j}\right)$ is the wave propagation factor from the scatterer at $\vec{r}_{j}$ to the position $\vec{r}$, and $A_{0}\left(\vec{r}_{j}\right)$ is the amplitude of the incident wave. In a 2D case, $p(\vec{r}) \propto|\vec{r}|^{-1 / 2} \exp (i \vec{k} \vec{r})$ for the point scatterers [8]. For the objects of a finite extent the scattering is described by the more complicated Mie theory (see, e.g., Ref. [9]), which does not lead to analytical estimations. Considering the intermediate propagation from $\vec{r}_{j-1}$ to $\vec{r}_{j}$ in secondary

reflections, the corresponding reflected field is calculated as $\sum_{j} s^{2} A_{0}\left(\vec{r}_{j-1}\right) p\left(\vec{r}-\vec{r}_{j}\right) p\left(\vec{r}_{j}-\vec{r}_{j-1}\right)$. Since $p\left(\vec{r}_{j}-\vec{r}_{j-1}\right)=$ $p_{1}$ is independent of the position in grating $j$, the latter sum represents a field in position $\vec{r}$ from a respectively shifted image source. Analogously the secondary reflections from all other scatterers are to be calculated, resulting in correspondingly shifted image sources.

Next, we estimate the magnitude of the effect. The spatial shape of the image source, considering the primary and all secondary sources, is

$$
A_{i m}(x)=s A_{0}(x)+s^{2} \sum_{n=1, \infty} p_{n}\left[A_{0}(x+n d)+A_{0}(x-n d)\right],
$$

with $p_{j}$ described by a Hankel function with the asymptotic $p_{j} \approx e^{i 2 \pi n d / \lambda}[\lambda /(n d)]^{1 / 2}[8]$.

The analysis of (1) is simpler in the transverse wave-number domain (i.e., the angular or the far-field domain) $A(x) \rightarrow$ $A\left(k_{x}\right),\left[A(x)=\int A\left(k_{x}\right) e^{i k_{x} x} d k_{x}\right]$ :

$$
A_{\text {im }}\left(k_{x}\right)=s A_{0}\left(k_{x}\right)\left[1+2 s \sum_{n=1, \infty} p_{n} \cos \left(k_{x} n d\right)\right],
$$

which gives the angular distribution of the coefficient of reflection [note that $k_{x}$, the transverse component of the wave vector, is a function of the incidence angle: $k_{x}=|k| \sin (\alpha)$ ]. The infinite sum over all neighbors in (1) and (2) converges and can be expressed by special functions [10], but, however, does not lead to analytically tractable results. In following we retain in (2) only the scattering from the nearest neighbors $n=1$. First, the secondary scattering due to nearest neighbors dominates: $\left|p_{1}\right|>\left|p_{2}\right|,\left|p_{3}\right|, \ldots$; second, the analysis of the complete sum (2) results in the same qualitative results, at least in the range of our interest $\lambda / 2<d<\lambda[10]$.

We note that Eq. (2) provides an alternative interpretation of beam focusing considering the process in angular (or the far-field) domain. Figure 1 interprets the focusing due to the appearance of the phase curvature of the image directly, in a space domain. Equation (2) considering only nearest-neighbor scattering simplifies to $r\left(k_{x}\right)=s\left[1+2 s p_{1} \cos \left(k_{x} d\right)\right]$. As the propagation factor $p_{1}$ is a complex value, then the phase of the reflection coefficient becomes angle dependent, showing asymptotically the parabolic function for small angles $k_{x}$. The parabolic angular dependence of the phase in an angular domain is equivalent to the parabolic dependence of phase in space domain (for Gaussian beams) and also indicates the focusing.

In the following we consider an incident Gaussian beam $A_{0}(x)=e^{-x^{2} / \Delta x_{0}^{2}}$ with the transverse distribution in the angular spectrum $A_{0}\left(k_{x}\right)=\Delta x_{0} / \sqrt{2} e^{-k_{x}^{2} / \Delta k_{0}^{2}}\left(\Delta k_{0} \Delta x_{0}=2\right)$. Expanding (2) around the propagation axis (directed normally to the grating) $k_{x} \ll|k|$, we obtain in the frame of a Gaussian beam approximation:

$$
A_{i m}\left(k_{x}\right) \approx \operatorname{sexp}\left[-k_{x}^{2}\left(\frac{1}{\Delta k_{0}^{2}}+s d^{2}\left|p_{1}\right| e^{i 2 \pi d / \lambda}\right)\right],
$$

which is valid for small angles $k_{x} \ll k$, and for weak reflection coefficients $s \ll 1$. 
Diffractive propagation of waves in homogeneous media results in a parabolic shift of the phases of the angular wave components: $\varphi\left(k_{x}\right)=-l k_{x}^{2} /\left(2 k_{0}\right)$ within the paraxial (parabolic) wave propagation theory. The reflection (3) is, therefore, equivalent to the propagation of the beam in free space over the distance:

$$
l_{\text {refl }}=2 k_{0} s d^{2}\left|p_{1}\right| \sin (2 \pi d / \lambda),
$$

which for $\lambda / 2<d<\lambda$ becomes negative. The $-l_{\text {reft }}$ therefore has a physical sense of a focal distance of the focusing lens. The maximum focal distance occurs at $d \approx 3 \lambda / 4$ and reaches the value $-l_{\text {reft }} \approx s \lambda\left|p_{1}\right| 9 \pi / 4$, as follows from (4). This estimates that the focal distance $f=-l_{\text {reft }}$ can reach the values of several wavelengths so that the effect can be measurable for sufficiently narrow beams.

In addition to the above discussed phase transformations of angular beam components (which is equivalent to the diffractive propagation of the beam), the narrowing and broadening of the angular spectrum also follow from the Gaussian beam approach (3). In a coordinate space, the latter represents the diffusion or antidiffusion of the beam, depending on the phase difference between the primary and secondary image sources. Inspection of (3) yields the minimum of the width of focused beam:

$$
\Delta x^{2}=\Delta x_{0}^{2}+4 s d^{2}\left|p_{1}\right| \cos (2 \pi d / \lambda),
$$

which for $d \approx \lambda / 2$ gives an estimation $\Delta x^{2}=\Delta x_{0}^{2}-s\left|p_{1}\right|$, which is also a measurable effect for sufficiently narrow beams.

Next we calculate the most relevant characteristics of the beam reflected from a grating applying the multiple scattering theory (1). The results are shown in Fig. 2, which summarizes the focal distance and the half-width of the reflected beam in a focal plane depending on frequency. The half-width of a reflected beam in a particular parameter range indeed becomes

(a)

(b)
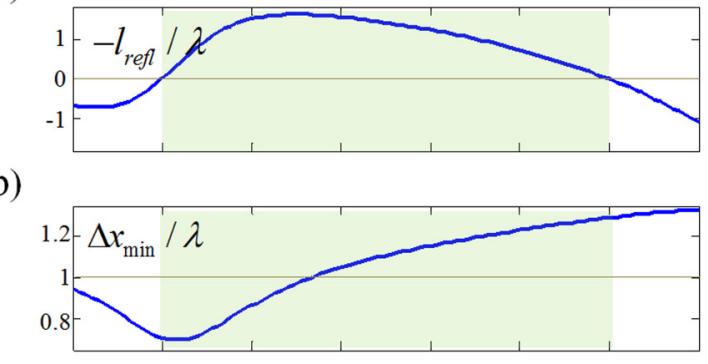

(c)

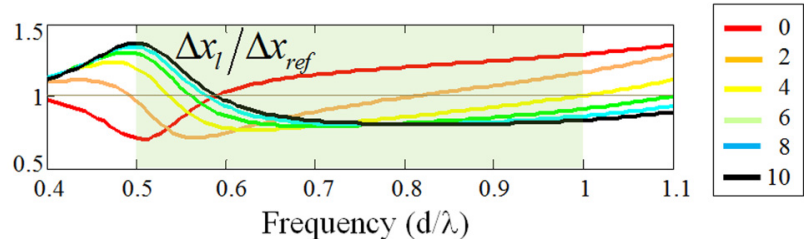

FIG. 2. (Color online) (a) The normalized focal lengths, (b) the normalized minimum width of the focussed beam (at the waist), and (c) the width of the beam for different propagation distances $l$ from 0 to $10 \lambda$ ( $l$ is the total propagation distance from the source to the grating and from grating to detecting plane) vs the normalized grating period. Half-width of the incident beam is $\Delta x_{0}=1.0 \lambda$, primary reflection coefficient is $s=0.25$, and $\left|p_{1}\right|=1,\left|p_{2}\right|,\left|p_{3}\right|, \ldots=0$.
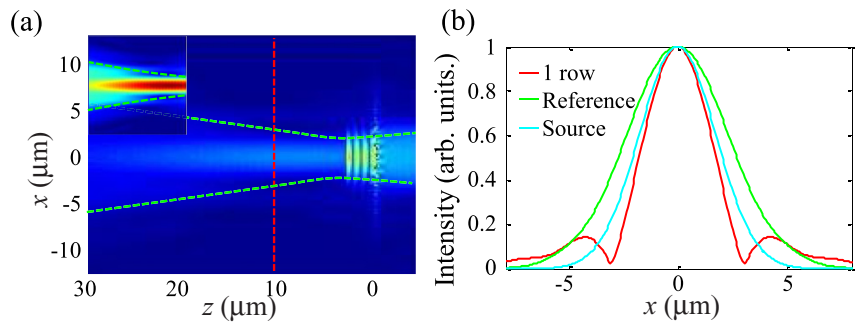

FIG. 3. (Color online) (a) Intensity distributions of the field for TE polarized radiation. The inset shows the intensity distribution of reflected field only. (b) The transverse beam profiles at a particular plane (indicated by vertically red dashed line). The boundaries of the reference beam (reflecting from the flat interface in the same position) are shown by two green dashed lines for comparison (at $e^{-2}$ intensity level).

smaller than that of the reference beam, which indicates the focusing effect.

Most importantly, the minimum width of the reflected beam can become even smaller than the width of the initial beam. This indicates that a subwavelength grating can show a real focusing rather than imaging such as by flat photonic-crystal lenses. A flat lens can restore the initial width of the beam but cannot focus the beam to a smaller focal spot than the source beam itself.

\section{FINITE DIFFERENCE TIME DOMAIN (FDTD) ANALYSIS}

In order to demonstrate the phenomenon in real systems, exact numerical simulations of the beam reflection and propagation were performed by using the FDTD approach. The typical space distributions of the reflected fields are given in Fig. 3. The source is positioned at a fixed distance of $3 \mu \mathrm{m}$ in front of the subwavelength grating as indicated in Fig. 3(a). The full beam width is considered to be $5 \mu \mathrm{m}$, which corresponds to the Rayleigh distance of $12 \mu \mathrm{m}$ at the frequency $f=$ $0.6(d / \lambda)$. The grating is made of dielectric cylinders with a reflective index of $n=3$ in an air background of $n=1$. The grating period $d$ is $1 \mu \mathrm{m}$, and the radius of cylinder is $r=$ $0.2 d$. The intensity distribution of the back-propagating beam shows clear signatures of focusing for the TE polarization (the electric field is normal to the plane of incidence, i.e., is parallel to the axis of cylindrical scatterers). For the TM polarization, the effect of focusing is absent or very weak, as the secondary reflections (the scattering) at $90^{\circ}$ approach zero for this TM polarization.

The FDTD calculations yield that the focal distances are slightly larger than those analytically estimated, reaching values of around $10 \mu \mathrm{m}$ for the given set of parameters. The width of the reflected beam is obtained smaller than that of the reference beam in a measurable parameter range, as expected. We also could find a range of the parameters (centered around the optimum period of the grating $d=0.6 \lambda$ ) where the reflected beams in focus are slightly narrower than the incident beam. The reflected energy varies from $20 \%$ to $40 \%$ of the initial energy depending on the parameters; under optimum focusing conditions shown in Fig. 3 the reflected energy is $27 \%$. 
(a)

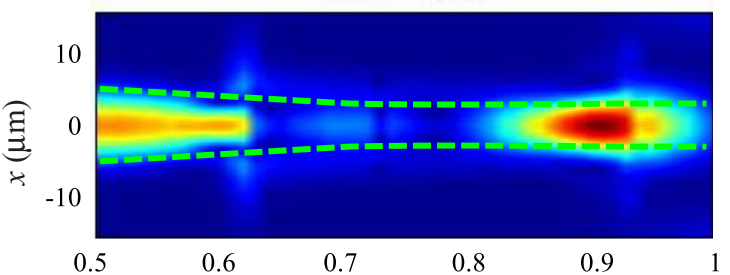

(b)

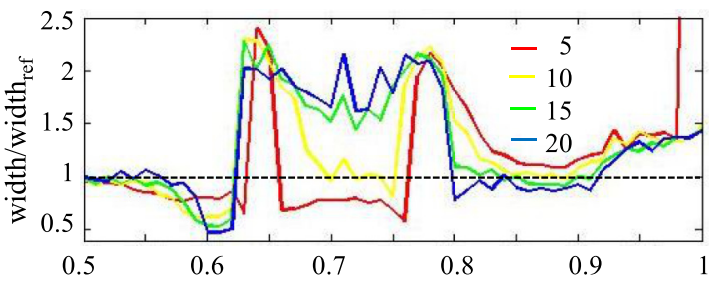

(c)

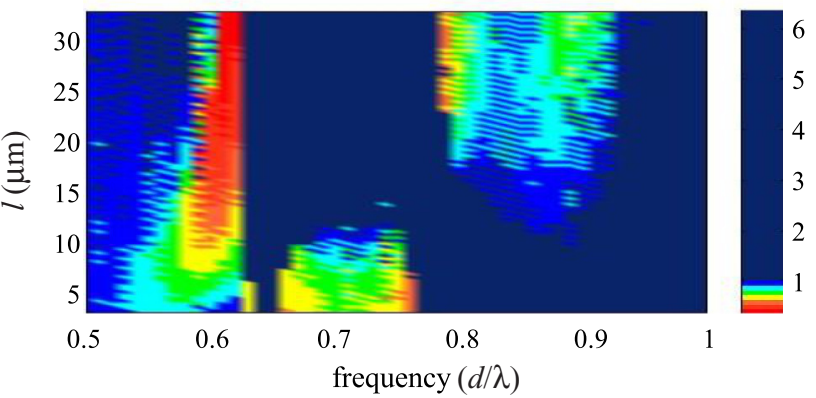

FIG. 4. (Color online) Summary of the quantitative performance of the focusing. (a) Map of transverse distribution of intensity of reflected beam at a distance $10 \mu \mathrm{m}$ in front of the grating, depending on frequency. The width of the reference beam is shown by dashed lines. (b) The width of the reflected beam (normalized by the width of reference beam) depending on frequency at different propagation distances $l$ from the grating. (c) The dependence of beam width (normalized to the width of the reference beam) on frequency (horizontal) and propagation distance $l$ (vertical).

The quantitative performance of the focusing is summarized in Fig. 4, where the width of the focused beam versus frequency is represented, in good correspondence with analytical estimations [Fig. 2(c)].

\section{FOCUSING BY TWO-DIMENSIONAL GRATINGS}

Furthermore, we extended our analysis to the 3D case studying reflection of the axisymmetric Gaussian beam from the $2 \mathrm{D}$ grating. The semianalytic calculations in the $3 \mathrm{D}$ case are performed by superposing the shifted images of sources depending on the symmetry of the lattice, in a similar way as in the $2 \mathrm{D}$ case. For the square lattice the composed image is the following:

$$
\begin{aligned}
A_{i m}(x, y)= & s A_{0}(x, y) \\
& +s^{2} \sum_{n=1, \infty} p_{n}\left[A_{0}(x+n d, y)+A_{0}(x-n d, y)\right] \\
& +s^{2} \sum_{n=1, \infty} p_{n}\left[A_{0}(x, y+n d)+A_{0}(x, y-n d)\right]
\end{aligned}
$$

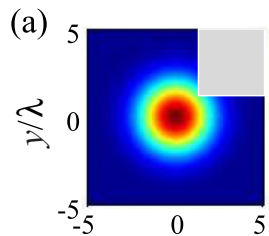

(b)
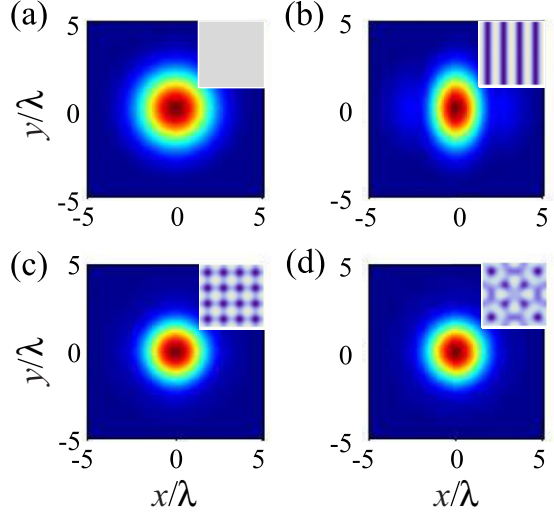

(d)

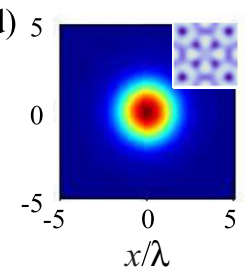

(e)

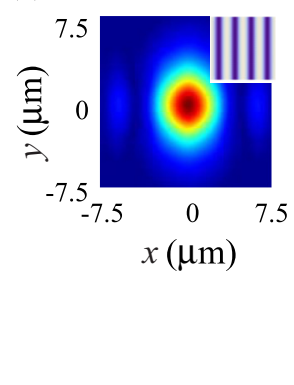

FIG. 5. (Color online) Reflections of a Gaussian beam from a 2D grating of different symmetries as obtained by semianalytic study [Eq. (6)]. (a) The reference beam (reflection from the flat surface) for comparison; (b) one-dimensional periodic array of cylinders; (c) periodic grating of square symmetry; (d) quasiperiodic grating of octagonal symmetry, where the intensity distributions are calculated at $5.5 \lambda$ in front of grating; (e) intensity distributions calculated by FDTD with the same parameters $\left(\Delta x_{0}=1.0 \lambda, r=0.2 d, d=0.6 \lambda\right)$.

The results are summarized in Fig. 5. For the grating in the form of parallel cylinders, the focusing occurs only along the grating direction, whereas the diffractive broadening in the vertical direction coincides with that of the reference beam. The elliptic shape of the spot, as shown in Fig. 5(b), indicates the focusing by subwavelength grating in one quadrature. For grating of square or of octagonal symmetry, the focusing occurs in both quadratures [Figs. 5(c) and 5(d)]. The focused beam in Fig. 5(c) shows a weak (hardly visible) square symmetry, which is due to the square symmetry of the grating. The grating of higher order symmetries, e.g., hexagonal or octagonal [see Fig. 5(d)], results in perfectly isotropic focusing. The exact 3D calculations using the FDTD method, presented in Fig. 5(e), prove the analytical predictions, as follows from comparison with Fig. 5(b).
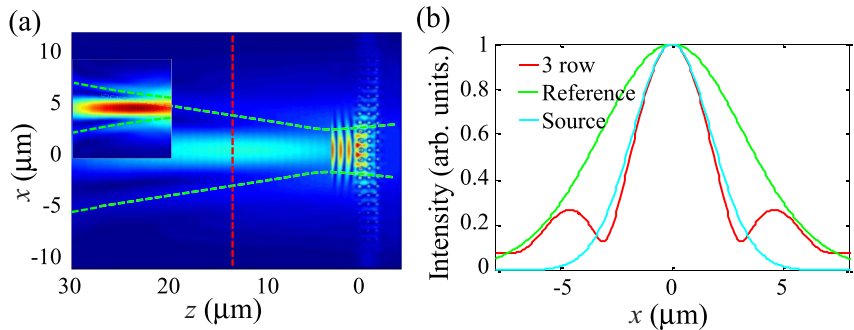

FIG. 6. (Color online) (a) Intensity distributions of the reflected beam from 3 rows of scatterers as obtained by FDTD simulations. The inset shows the intensity distributions of reflected field only. (b) The transverse beam profiles at particular (indicated by vertically red dashed line) plane. The boundaries of the reference beam are shown by two green dashed line for comparison. The rows are separated by $\lambda / 2$ in the $z$ direction. The other parameters and conditions are as in Fig. 3. 


\section{FOCUSING WITH SEVERAL PARALLEL ARRAYS}

The reflectivity from one array of scatterers ranges between $20 \%$ and $40 \%$ under optimum focusing conditions. However, the reflectivity, and the focusing performance in general, can be enhanced using several parallel arrays of scatterers (or several parallel planes of gratings in a $3 \mathrm{D}$ case). The strongest reflection is observed when the arrays are arranged in a configuration following the Bragg reflection condition in a longitudinal direction (for separation between arrays $d_{z} \approx \lambda / 2$ ). Our numerical calculations show enhancement of the effect: the increase of the focal distance, as well as the increase of reflectivity to $71 \%$, in this particular case. Figure 6 shows a case where a single grating (from Fig. 3 ) is substituting by an array of three gratings separated by $d_{z} \approx \lambda / 2$ in a longitudinal direction.

\section{CONCLUSIONS}

Summarizing, we predict and describe a physical phenomenon: beam focusing in reflections from flat subwavelength diffraction gratings. We estimate the parameters by a semianalytic multiple scattering technique, and by exact FDTD numerical calculations, and show that the effect is observable in optical systems. The phenomenon could also be observable in other wave systems, such as sound waves reflecting from acoustic gratings, for surface plasmon polariton waves on periodically corrugated surfaces on a subwavelength scale, or from subwavelength period gratings of metallic nanowires (probably the effect enhanced by plasmonic resonances in the latter cases). However, the focusing effect is demonstrated only by arrays of dielectric cylinders in this paper.
The effect is also possible in transmissions, which follows from the analytical expressions Eqs. (1)-(3), and which follows from our FDTD simulations (not presented). However, the effect in transmissions is weaker than in reflections, as it relies on the interplay between the zero order transmission of $O(1)$ and secondary reflections of $O\left(s^{2}\right)$. In reflections the interplay between the first order reflection of $O(s)$ and the secondary reflections of $O\left(s^{2}\right)$ of more similar orders of magnitude is at the root of more pronounced focusing effect.

One of the potential applications of the proposed effect is a design of Fabri-Perrot microresonators with zero or negative diffraction (equivalently with zero or negative equivalent length). It is known that the zero or negative diffraction of the resonators can be achieved by (fully or partially) filling the resonator by metamaterials with negative refraction [11] or by photonic crystals providing negative diffraction [12]. Such a configuration in particular can support spatial solitons for defocusing nonlinearity [13]. The subwavelength diffraction gratings, serving as focusing mirrors of the resonator, could be also implemented in order to modify the diffraction properties of the resonators and to support the cavity solitons in cavities filled by nonlinear media, among others.

We note finally that the proposed focusing occurs at a normal incidence (and normal reflection) from the grating. A similar effect, the beam focusing from flat chirped dielectric mirrors, recently demonstrated in Ref. [14], occurs for large incidence angles of the beams and never at a normal incidence.

\section{ACKNOWLEDGMENT}

The work is financially supported by Spanish Ministerio de Educación y Ciencia and European FEDER through projects FIS2011-29734-C02-01 and -02.
[1] H. F. Ghaemi, T. Thio, D. E. Grupp, T. W. Ebbesen, and H. J. Lezec, Phys. Rev. B 58, 6779 (1998).

[2] F. Ramos-Mendieta and P. Halevi, Phys. Rev. B 59, 15112 (1999).

[3] E. C. Le Ru, E. Blackie, M. Meyer, and P. G. Etchegoin, J. Phys. Chem. 111, 13794 (2007).

[4] F. Brückner, D. Friedrich, T. Clausnitzer, M. Britzger, O. Burmeister, K. Danzmann, E.-B. Kley, A. Tünnermann, and R. Schnabel, Phys. Rev. Lett. 104, 163903 (2010).

[5] E. Hecht, Optics, 4th ed. (Addison Wesley, New York, 2002).

[6] L. Verslegers, P. B. Catrysse, Zongfu Yu, J. S. White, E. S. Barnard, M. L. Brongersma, and Shanhui Fan, Nano Lett. 9, 235 (2008).

[7] D. Fattal, J. Li, Z. Peng, M. Fiorentino, and R. G. Beausoleil, Nature Photonics 4, 466 (2010).

[8] H. C. van de Hulst, Light Scattering by Small Particles (Dover, New York, 1981).
[9] J. H. Seinfeld and S. N. Pandis, Atmospheric Chemistry and Physics, 2nd ed. (John Wiley and Sons, New York, 2006).

[10] The infinite sum (2), with asymptotic expressions for propagation coefficients $p_{j} \approx e^{i 2 \pi d n / \lambda}[\lambda /(n d)]^{1 / 2}$ converges everywhere, except singular point $d=\lambda$ (generally $d=n \lambda$ ), and can be expressed in terms of special (polylog) functions. The series expansions of the obtained analytical expression shows the same qualitative behavior as (3), i.e., when considering the scattering between nearest neighbors only.

[11] P. Tassin, G. Van der Sande, I. Veretennicoff, P. Kockaert, and M. Tlidi, Opt. Express 17, 9428 (2009).

[12] R. Iliew, C. Etrich, T. Pertsch, F. Lederer, and K. Staliunas. Opt. Lett. 33, 2695 (2008).

[13] K. Staliunas, O. Egorov, Yu. S. Kivshar, and F. Lederer, Phys. Rev. Lett. 101, 153903 (2008).

[14] Y. C. Cheng, M. Peckus, S. Kicas, J. Trull, C. Cojocaru, R. Vilaseca, R. Drazdys, and K. Staliunas, Phys. Rev. A 87, 045802 (2013). 